\title{
DIII-D PLASMA CONTROL SIMULATION ENVIRONMENT
}

\author{
by \\ J.A.LEUER, R.D. DERANIAN, J.R. FERRON, \\ D.A. HUMPHREYS, R.D. JOHNSON, B.G. PENAFLOR, \\ M.L. WALKER, A.S. WELANDER, R.R. KHAYRUTDINOV, \\ V. DOKOUKA, D.H. EDGELL, and C.-M. FRANSSON
}




\section{DISCLAIMER}

This report was prepared as an account of work sponsored by an agency of the United States Government. Neither the United States Government nor any agency thereof, nor any of their employees, makes any warranty, express or implied, or assumes any legal liability or responsibility for the accuracy, completeness, or usefulness of any information, apparatus, product, or process disclosed, or represents that its use would not infringe privately owned rights. Reference herein to any specific commercial product, process, or service by trade name, trademark, manufacturer, or otherwise, does not necessarily constitute or imply its endorsement, recommendation, or favoring by the United States Government or any agency thereof. The views and opinions of authors expressed herein do not necessarily state or reflect those of the United States Government or any agency thereof. 
GA-A24470

\title{
DIII-D PLASMA CONTROL SIMULATION ENVIRONMENT
}

\author{
by \\ J.A.LEUER, R.D. DERANIAN, J.R. FERRON, \\ D.A. HUMPHREYS, R.D. JOHNSON, B.G. PENAFLOR, \\ M.L. WALKER, A.S. WELANDER, R.R. KHAYRUTDINOV," \\ V. DOKOUKA, ${ }^{*}$ D.H. EDGELL, ${ }^{\dagger}$ and C.-M. FRANSSON \\ ${ }^{*}$ TRINTI Laboratory \\ tFarTech, Inc. \\ $\ddagger$ Chalmers University
}

This is a preprint of a paper presented at the 20th IEEE/NPSS Symposium on Fusion Engineering, San Diego, California, October 14-17, 2003 and to be published in Fusion Science and Technology.

\author{
Work supported by \\ the U.S. Department of Energy \\ under Contract No. DE-AC03-99ER54463
}




\title{
DIII-D Plasma Control Simulation Environment
}

\author{
J.A. Leuer, R.D. Deranian, J.R. Ferron, D.A. Humphreys, R.D. Johnson, B.G. Penaflor, M.L. Walker, A.S. Welander,

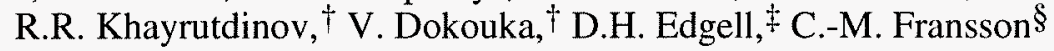 \\ General Atomics, P.O. Box 85608, San Diego, California 92186-5608 \\ †TRINITI Laboratory \\ $\ddagger$ Fartech, San Diego, California 92121 \\ $\$$ Chalmers University
}

\begin{abstract}
Many advances have been made to the DIII-D plasma control simulation environment since the previously developed hardware-in-the-loop plasma shape simulation capability was reported. In the present paper we summarize the major improvements to this simulation environment, including, introduction of the non-linear plasma evolution code DINA. Comparisons with DIII-D experimental results are presented. Recent model developments in advanced neoclassical tearing mode (NTM) and resistive wall mode (RWM) control are presented.
\end{abstract}

\section{INTRODUCTION}

The DIII-D experimental program is rapidly evolving toward development of sustainable Advanced Tokamak (AT) plasma configurations [1]. The great flexibility of the DIII-D machine, with its 18 close fitting poloidal field control coils, $20 \mathrm{MW}$ of neutral beam injection, $6 \mathrm{MW}$ of electron cyclotron heating, and extensive set of diagnostics, makes this machine a leader in AT plasma development. Critical to DIII-D's AT mission is internal plasma profile control. This requires accurate modeling tools, flexible real-time control architecture and advanced control methodologies. Over the years General Atomics has continued to develop state-of-the-art tools required to meet the objectives of our AT based mission. This paper summarizes several aspects of our model-based advanced control effort, which have been evolving since last reported [2].

A complete suite of design, analysis and simulation tools has been developed to better implement real time controllers for AT operation in DIII-D [3]. The collection of software consists of model-based algorithms to simulate DC power supplies, fast switched power supplies, field shaping and Ohmic heating coils, passive vacuum vessel, linear plasma response, data filters, magnetic diagnostics and A/D and D/A signal converters [2]. The validated algorithms provide a foundation for model-based controller design and are used in combination to build a complete simulator of the DIII-D machine for model and controller validation. This simulation environment, in conjunction with the flexible software architecture implemented in the plasma control system (PCS) [4], allows for a complete software simulation of the plasma shape control system using the DIII-D simulator as a hardware-in-the-loop component and provides the ability to test and validate newly developed control algorithms without requiring costly experimental time [2].

In addition to the machine component models, a complete set of controller design tools has been developed to implement advanced real-time control algorithms. An example of their uses is in the development and implementation of multi-inputmulti-output (MIMO) shape controllers for better plasma shape control [5]. The tokamak analysis tools are implemented in the MATLAB/SIMULINK ${ }^{\mathrm{TM}}$ computational environment, which provides a rich assortment of numerical algorithms for simulation and controller development, and the ability to interface with other languages such as C and FORTRAN. All tools in our tokamak analysis and control suite are modelbased, making them applicable over a wide range of system parameters and to other toroidal devices.

This paper describes recent improvements to this analysis environment. In particular, the non-linear axisymmetric, timedependent plasma simulation code, DINA [6] has been implemented within the environment to augment our existing linear plasma response model [7]. Preliminary validation of the model will be presented. Recent progress in controller development for NTM suppression based on detailed simulation is presented. Finally, recent progress in RWM detection algorithm development is summarized.

\section{DINA IMPLEMENTATION}

The DINA code [6] is an axisymmetric time dependent equilibrium evolution code. It contains many models for relevant tokamak subsystems including $\mathrm{PF}$ circuits, energy transport, neutral beam injection, electron cyclotron heating, current drive, bootstrap currents and $\alpha$-particle heating. It has many advantages over the linear model [7] used in the past, including non-linear response, internal plasma profile evolution, and numerous plasma-actuator interaction modules. In the present implementation, only the plasma evolution aspects of the code and profile modifications associated with electron cyclotron current drive (ECCD) are utilized within our environment. This implementation allowed utilization of the model in the design of algorithms for NTM suppression using ECCD deposition.

The DINA code is written in FORTRAN and is implemented within our MATLAB/SIMULINK ${ }^{\text {TM }}$ environment using a "MEX S-function". A similar implementation has been used in the analysis of TCV for simulation of electron cyclotron heating and current drive [8]. In our implementation, the machine and plasma functions are handled separately, following a block-oriented modeling philosophy.

The PF-coil circuit equations are constructed and solved separately from the plasma evolution equations, which are handled within DINA. An example of separation of the coil system from the plasma system is shown below from the circuit representation of the combined $\mathrm{PF} /$ plasma system [7]:

$$
\begin{gathered}
M_{s s} \frac{d I_{s}}{d t}+R_{s} I_{s}+\frac{\partial \psi_{s}}{\partial z_{p}} \frac{\partial z_{p}}{\partial I_{s}} \frac{d I_{s}}{d t}+\frac{\partial \psi_{s}}{\partial r_{p}} \frac{\partial r_{p}}{\partial I_{s}} \frac{d I_{s}}{d t}+M_{s p} \frac{d I_{p}}{d t}=V_{s} \\
L_{p} \frac{d_{p}}{d t}+R_{p} I_{p}+\frac{\partial \psi_{p}}{\partial r_{p}} \frac{d_{p}}{\partial I_{s}} \frac{d I_{s}}{d t}+\frac{\partial \psi_{p}}{\partial z_{p}} \frac{d z_{p}}{\partial I_{s}} \frac{d I_{s}}{d t}+M_{p s} \frac{d I_{s}}{d t}=0
\end{gathered}
$$


This can be written in a block-oriented feedback mode for the device specific dynamics of the PF-coil system as:

$$
\frac{\mathrm{d} \mathrm{I}_{\mathrm{s}}}{\mathrm{dt}}=-\mathrm{M}_{\mathrm{ss}}^{-1} \mathrm{R}_{\mathrm{s}} \mathrm{I}_{\mathrm{s}}+\mathrm{M}_{\mathrm{ss}}^{-1} \mathrm{~V}_{\mathrm{s}}+\mathrm{M}_{\mathrm{ss}}^{-1} \mathrm{~V}_{\text {loop }}
$$

and for the plasma block dynamics as:

$$
\begin{aligned}
& \frac{\mathrm{dI}_{\mathrm{p}}}{\mathrm{dt}}=-\mathrm{L}_{\mathrm{p}}^{-1} \mathrm{R}_{\mathrm{p}} \mathrm{I}_{\mathrm{p}}-\mathrm{L}_{\mathrm{p}}^{-1}\left(\frac{\partial \psi_{\mathrm{p}}}{\partial \mathrm{r}_{\mathrm{p}}} \frac{\mathrm{dr} \mathrm{r}_{\mathrm{p}}}{\partial \mathrm{I}_{\mathrm{s}}}+\frac{\partial \psi_{\mathrm{p}}}{\partial z_{\mathrm{p}}} \frac{\mathrm{dz} \mathrm{p}_{\mathrm{p}}}{\partial \mathrm{I}_{\mathrm{s}}}+\mathrm{M}_{\mathrm{ps}}\right) \frac{\mathrm{dI}_{\mathrm{s}}}{\mathrm{dt}} \\
& V_{\text {loop }}=-M_{s p} \frac{d I_{p}}{d t}-\left(\frac{\partial \psi_{s}}{\partial z_{p}} \frac{\partial z_{p}}{\partial I_{s}}+\frac{\partial \psi_{s}}{\partial r_{p}} \frac{\partial r_{p}}{\partial I_{s}}\right) \frac{d I_{s}}{d t}
\end{aligned}
$$

where: $\mathrm{I}$ is current, $\mathrm{V}$ is voltage, $\mathrm{M}$ and $\mathrm{L}$ are inductances, $\mathrm{R}$ is resistance, $\psi$ is flux, $r \& z$ are plasma displacements and $t$ is time. Subscripts $s$ and $p$ correspond to the coil system and plasma, respectively and $V_{\text {loop }}$ is the voltage on each coil from the plasma. In the above equations variations in the plasma internal parameters like $\beta_{\mathrm{p}}$ and $l_{1}$ have been neglected.

The DINA module replaces the plasma response functions in the above formulation where the input/output functions of the DINA block are the PF-coil current states $\left(\mathrm{I}_{\mathrm{S}}\right)$ and the voltage imposed at the coils $\left(\mathrm{V}_{\text {loop }}\right)$ the plasma, respectively. Fig. 1 shows the SIMULINK ${ }^{\mathrm{TM}}$ implementation in the DIII-D simulation environment [2]. At the top level the DINA plasma/circuit model is plug compatible with the linear plasma response model and linear or non-linear simulation can be accomplished by switching this block.

The DINA plasma/circuit model in Fig. 1 is expanded in detail in Fig. 2. The connection between the S-function, DINA block and the DIII-D simulation environment is through direct-feed and feed-back loops between the DIII-D circuit model and DINA plasma evolution model. The circuit model consists of the state-space description of the poloidal field (PF) and vaccum vessel (VV) conductors, assembled and con- strained based on a particular DIII-D discharge configuration. Coil currents from the state-space model are passed to the DINA model. These currents, along with the conductor to plasma influence matrix, provide DINA with external magnetic perturbations in the plasma. Conversely, the DINA module computes plasma voltages at each conductor and feeds this back to the circuit model for combination with the power supply voltages. Diagnostic signal outputs consist of the sum of PF, vessel and plasma contributions.

\section{DINA COMPARISON WITH DIII-D EXPERIMENTS}

Comparisons between the simulator output and DIII-D experimental results show good agreement for the discharges studied. In performing the comparisons the system is initialized using an EFIT [9] reconstruction of the plasma from DIII-D diagnostic data. The overall system is unstable to vertical motion and must be stabilized with a feed back loop when running in open loop and using DIII-D data as input. This feed back loop is shown around the DINA/circuit module in Fig. 1 and its parameters are set to those used in the experiment. When operating the simulator in closed loop feed back with the PCS this loop is disabled and the PCS provides stabilization. Within the DINA model the plasma evolution includes magnetic diffusion and the initial resistivity is set to match Ohmic dissipation during the discharge. The DIII-D actuator data (PF power supply commands from the plasma control system) from shot archives is input to the simulator (Port: "(1) Command In" in Fig. 1). The response of the simulator to the digital inputs is output at output port: "(3) Diagnostic Out" and recorded for comparison with actual discharge data.

Fig. 3 shows a typical comparison for a moderate beta, double-null discharge (shot 99652). In this shot there is a preprogrammed rigid radial shift in the plasma from 3.5 to $4.5 \mathrm{~s}$. The plasma is controlled with a $+5 \mathrm{~cm}$ to $-2 \mathrm{~cm}$

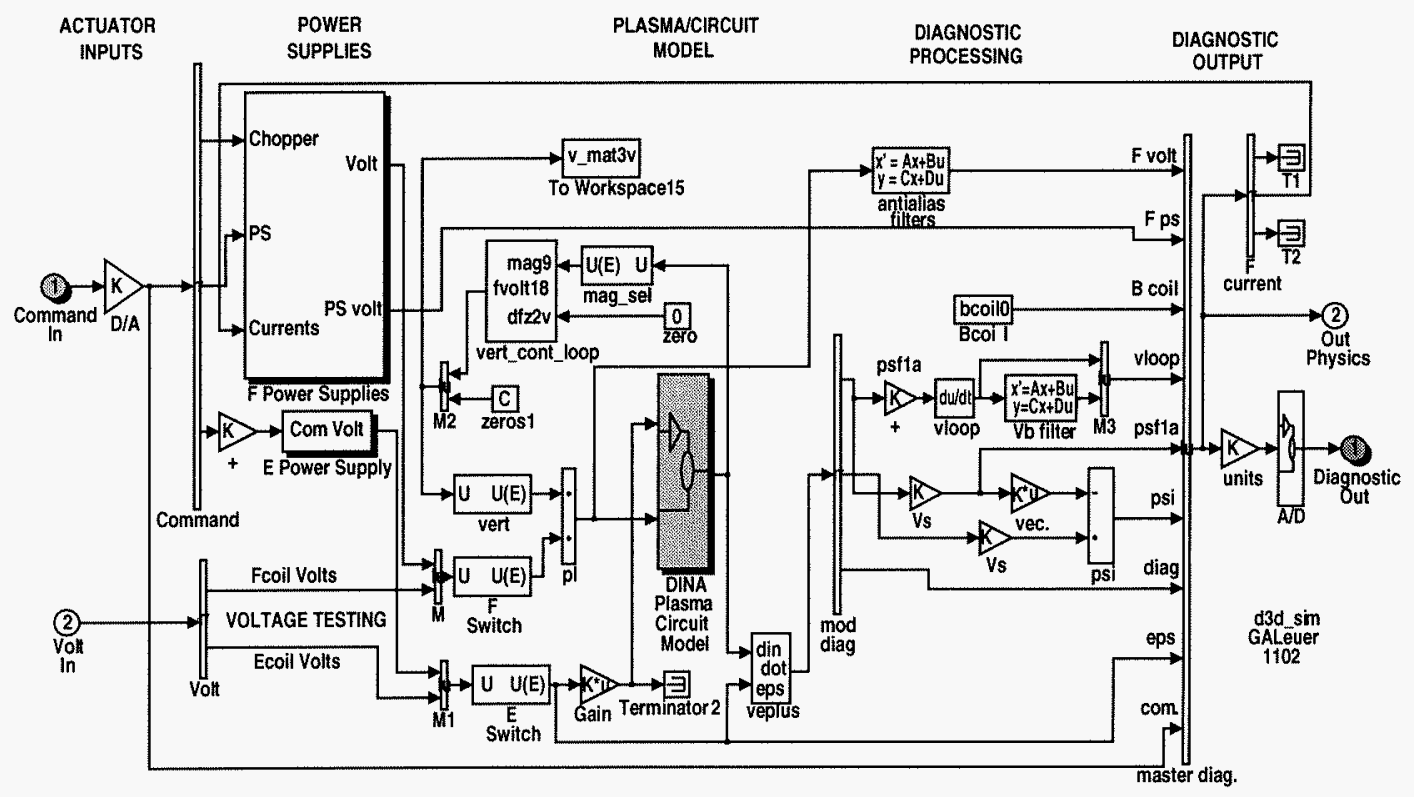

Fig. 1. Schematic of the DINA-DIII-D simulator showing major components. "Command In" is power supply/chopper commands from PCS; "Diagnostics Out" is simulated diagnostic output to PCS. "DINA Plasma Model" is plug compatible with the DIII-D linear plasma model. 
J.A. LEUER, et al.

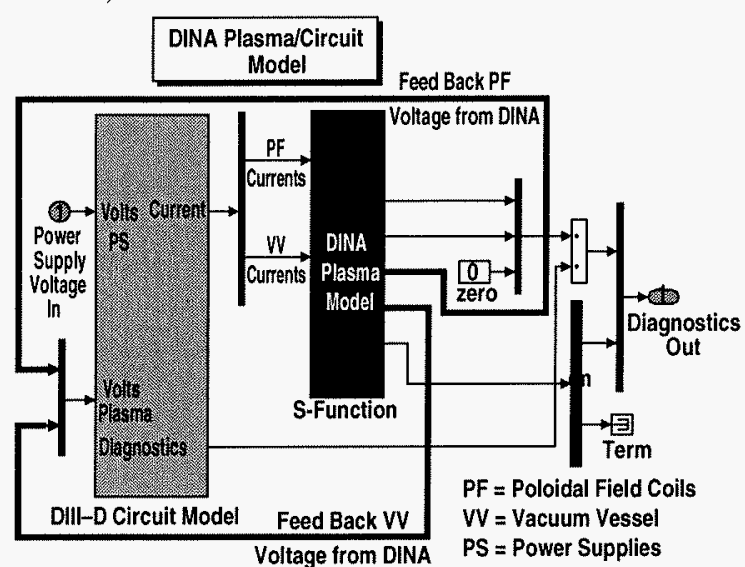

Fig. 2. Schematic of the DINA plasma/circuit model interface. DINA is implemented using a S-Function wrapper. Coil currents are input and plasma induced voltages on the coils is feedback output.

sawtooth waveform. The first trace in the figure shows a typical power supply input waveform; this represents input to the simulator. The remaining traces represent the typical diagnostic output. Ohmic heating and poloidal field coil currents, as delineated by ecoila and fla, respectively, show close agreement with the experimental results. Other diagnostics such as flux loops (psfla \& psf7b) and magnetic probes (mpil $1 \mathrm{~m} 322$ and mpi7fb322) also show reasonable agreement. Small divergence in the signal is partially due to the simple neo-classical magnetic diffusion model used in the analysis. In particular, starting after the $4 \mathrm{~s}$ one of the neutral beam drops out causing some decrease in beta which is not simulated in the present DINA/DIII-D implementation.

Fig. 4 shows $100 \mathrm{~ms}$ snapshots of the plasma shape as reconstructed from experimental data using EFIT and based on DINA predictions. The overall plasma excursion stops just before the outboard limiter position without contacting the wall. The DINA results are shown to agree reasonably well

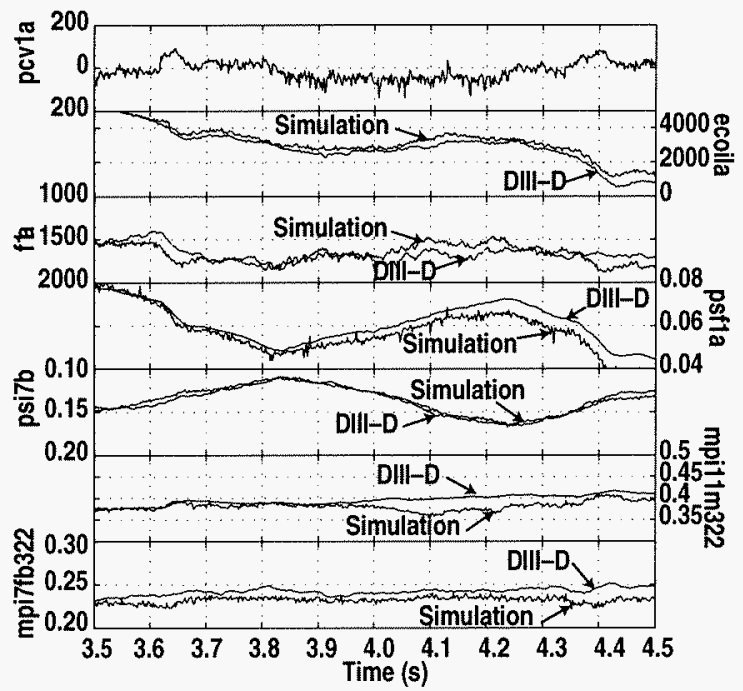

Fig. 3. Representative DINA/DIII-D simulator comparisons with experimental signals. Solid: experiment; dashed: simulator.
DIII-D PLASMA CONTROL SIMULATION ENVIRONMENT

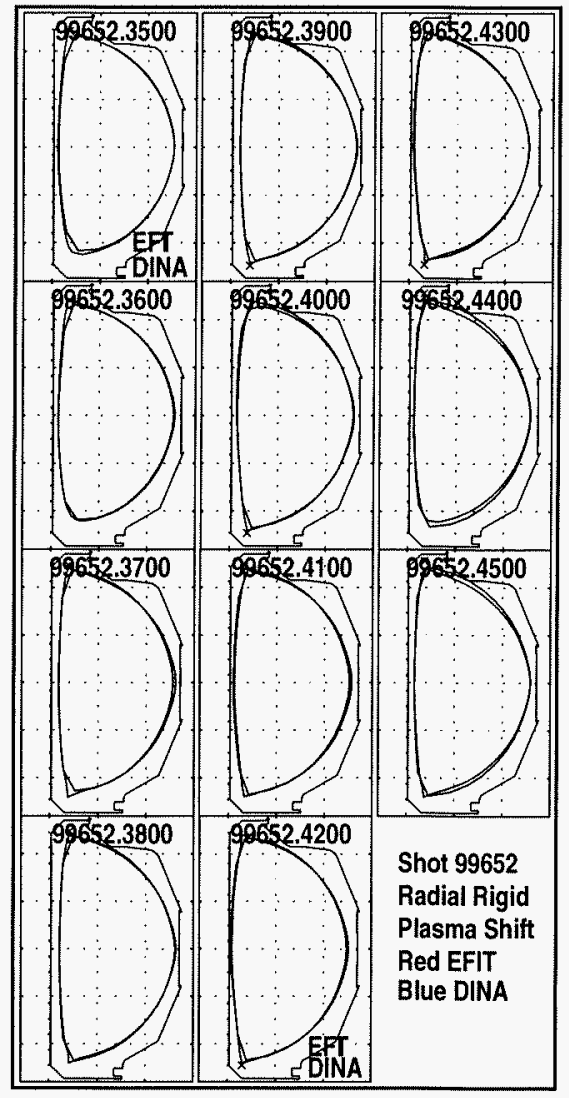

Fig. 4. Comparison of DINA predicted plasma shape to DIII-D shape reconstructed from experimental data using EFIT [9]. The plasma is rigidly moved in the radial direction during the 3.5 to 4.5 s test period.

with the experimental results. The radial motion of the plasma is very accurately simulated. Small variation in shape near the $\mathrm{X}$-point are, in part, due to a lower resolution grid used in DINA and small shape variations toward the end of the discharge are attributable to the previously discussed beam drop-out.

\section{MHD STABILITY CONTROL DEVELOPMENTS}

A number of models and control algorithms have been developed for active suppression of MHD instabilities including NTM and RWM modes.

The NTM is stabilized in DIII-D by application of ECCD at the island location to replace the missing bootstrap current which characterizes the mode. Since the location of the island cannot be directly measured in DIII-D in realtime, the optimal alignment is found through one of two search algorithms. The "Search and Suppress" algorithm performs a scan across a specified region by moving the plasma major radius or adjusting the toroidal field in discrete steps and monitoring the mode amplitude (which can be measured). The reduction in mode amplitude reflects the island size, and allows the controller to infer the degree of alignment, freezing the major radius or toroidal field at the optimal value until the mode is eliminated. An alternate approach, the "Target Lock" 
algorithm, estimates the location of the island based on the dynamic response of the mode amplitude as the plasma major radius or toroidal field is perturbed. The algorithm uses the Modified Rutherford Equation (MRE) [10] to model the predicted island response and infer its location from the observed dynamic response. Each discrete estimate produces a command for a new position or field value, and the resulting effect on the mode allows the Target Lock algorithm to further refine the estimate, eventually homing in on the correct alignment.

The proper function of these two algorithms requires careful selection of a set of parameters describing the nonlinear calculation and logic of the controllers. In order to determine the best choices for these parameters and test the function of the controllers, two different types of simulation are used. In one type of simulation, the MRE is used to describe the island evolution in response to applied ECCD depending on the degree of alignment. The current drive can be predicted using the DINA ECCD model, or taken from experiment. The DINA simulation allows full closed loop testing to optimize parameters. The second type of simulation uses experimental data from an actual NTM discharge to drive the control algorithm in open loop. This allows validation of the control algorithm, checking of units and diagnostic signals, and testing of the actual implementation in the PCS. Fig. 5 shows the result of such a simulation using data from a DIII-D discharge in which Search and Suppress was used to stabilize the 2/1 NTM by varying the toroidal field (Bt, shown in the lower frame). The mode amplitude history from the experiment is indicated in the upper frame as "Search and Suppress." Given the same data available from that discharge, the Target Lock algorithm produces the more rapid suppression history indicated. The "Maximum Suppression" trace in the figure is a theoretical best possible (simulated) suppression. The simulation thus indicates that the Target Lock algorithm should improve the suppression rate over that produced by the Search and Suppress, but would not achieve the maximum suppression rate possible which is produced by ideal alignment from the moment the control is enabled.

Several new RWM models and controllers have also been developed. Improved RWM control is produced by combining a static matched filter technique with a dynamic Kalman filter

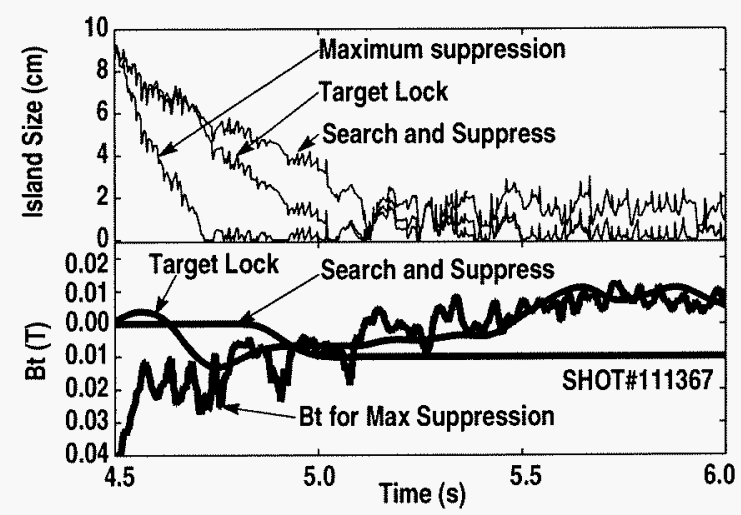

Fig. 5. NTM suppression simulation comparing Target Lock and Search \& Suppress algorithms using Bt control. The Maximum suppression is the field needed to maintain ECCD and the island surface perfectly aligned. to perform RWM mode identification and edge localized mode (ELM) rejection. Models and controllers were developed and validated against DIII-D experimental data. A complete closed loop simulation of the system shows good ELM rejection and RWM suppression. [11].

\section{CONCLUSIONS}

The DIII-D simulation environment has been enhanced with the addition of the non-linear plasma evolution code, DINA. A modular approach has been developed to allow introduction of any plasma model, independent of DIII-D system-specific algorithms. This technique allows for easy introduction of other plasma models and for application to other tokamak machines. Comparisons of the model with results of the DIII-D experiment show excellent agreement. The new models and controllers have been made for NTM and RWM suppression.

\section{ACKNOWLEDGMENT}

Work supported by U.S. Department of Energy under Contract No. DE-AC03-99ER54463.

\section{REFERENCES}

[1] D.A. Humphreys, et al., "Advanced tokamak operation using the DIII-D plasma control system," Proc. of the 22nd Symp. On Fusion Tech., Helsinki, Finland, 2002, to be published in Fusion Eng. and Design.

[2] J.A.Leuer, et al., "Development of a Closed loop simulator for poloidal field control in DIII-D", Proc. of the 18th IEEE/NPSS Symp. On Fusion Engineering, Albuquerque, New Mexico, (Institute of Electrical and Electronics, Inc. Piscataway, 1999), p.531-34.

[3] D.A. Humphreys, et.al., "Integrated plasma control for advanced tokamaks," these proceedings.

[4] J.R. Ferron, et al., "Flexible software architecture for tokamak discharge control systems,", Proc. 16th IEEE Symp. on Fusion Engineering, Champaign, Illinois (Institute of Electrical and Electronics Engineers, Inc., Piscataway, 1996), Vol. 2, p. 870.

[5] M.L. Walker, et al., "Multivariable shape control development on the DIII-D tokamak," Proc. 17th Symp. on Fusion Engineering, San Diego, California, (Institute of Electrical and Electronics Engineers, Inc., Piscataway, 1998) p. 556.

[6] R.R.Khayrutdinov, V.E.Lukash, J. Comput. Phys 109 (1993) 193.

[7] D.A. Humphreys, M.L. Walker, "Minimal plasma response models for design of high- $\beta_{p}$ tokamak dynamic shape and position controllers," to be submitted to Fusion Technology.

[8] D. Raju, et al., "DINA simulations of TCV electron cyclotron current drive and heating", 29th EPS Conference on Plasma Phys. and Contr. Fusion, Montreux, 17-21 June 2002.

[9] L.L. Lao, et al., Nucl. Fusion 25, 1611 (1985).

[10] Hegna, C.C., Callen, J.D., Phys. Pl. 4 (1997) 2940.

[11] C.M Fransson, et al., "Model validation, ELM discrimination, and high confidence RWM control in DIII-D," accepted for pub. in Phys. Pl., June 2003. 